\title{
Haller als Herr von Goumoens-le-Jux und Eclagnens
}

\author{
Von Heinz Balmer
}

Das erste Ziel des Berner Aristokraten des 18. Jahrhunderts war die Wahl in den Großen Rat. Dieser hieß auch Rat der Zweihundert, CC. Wer ihm angehörte und somit «der Burgern» war, dem standen die Staatsämter offen. Bei den Osterwahlen konnte ihm eine Landvogtei oder der Aufstieg in den Kleinen Rat zuteil werden. Jetzt, als «Ratsherr», konnte er zu einem der vier Venner oder zu einem der beiden Seckelmeister werden und rückte so den beiden Schultheißen immer näher, die als «regierender» und «stillstehender» abwechselnd das Steuer des Staates lenkten.

Haller wurde 1745 der Burgern. Schultheiß Isaak Steiger, ein Verwandter seiner ersten Frau, förderte seine Wahl. 1753 erhielt Haller das auf vier Jahre begrenzte Amt des Rathausammanns und 1758 für sechs Jahre die Salzdirektion in Roche, die einer Landvogtei gleichkam. Der Eintritt in den Kleinen Rat gelang ihm trotz neunmaliger Bewerbung nicht.

Neben dem Verlangen nach dem öffentlichen Dienst bewegte den Berner Patrizier die Sehnsucht, Herr eines Schlosses oder eines Grundbesitzes zu sein. Wem von Haus aus kein solches Erbe zufiel, dem wurde es nicht selten durch die Heirat innerhalb seines Standes zuteil.

Sowohl durch seine erste wie durch seine zweite Frau hätte Haller, obschon er nicht in dieser Absicht heiratete, einen derartigen Besitz erlangen können. Der Vater seiner ersten Frau Marianne, der Apotheker Samuel Wyß (1677-1755), war durch seine Frau, Maria von Diesbach, Herr zu Mathod und La Mothe bei Yverdon geworden. Maria war die einzige Tochter Wilhelm Axel von Diesbachs. Dagegen war Marianne nicht die einzige Tochter, sondern besaß drei jüngere Schwestern, Euphrosine, Rosine und Katharina. Diese jüngste (1718-1809) erbte die Herrschaft und brachte sie in die Familie Thormann.

Hallers zweite Frau, Elisabeth Bucher, war eine Tochter des Ratsherrn Hans Rudolf Bucher (1681-1742), der das Schloß Holligen bei Bern besaß. Sein gleichnamiger Vater hatte es 1675 den beiden Töchtern Johann Jakob Thormanns (1623-1674) abgekauft. Jener ältere Hans Rudolf Bucher (1640-1719) war damals Landvogt in Frienisberg und wurde später Ratsherr, Venner und Deutschseckelmeister. Sein Sohn brachte es bis zum Venner. Elisabeth Haller-Bucher starb vor ihrem Vater. Ihre Geschwister verkauften Holligen 1742 dem Gatten ihrer Kusine, Daniel Fellenberg, der aus bescheidenen Verhältnissen zu Reichtum, preußischem Adel und zum Venneramt aufstieg. Fellenbergs Tochter Jo- 
hanna Margarita heiratete 1741 Gabriel Herbort (1705-1783), der 1752-1758 Hallers Vorgänger als Salzdirektor war, 1759 in den Kleinen Rat eintrat und 1764 das Schloß übernahm ${ }^{1}$.

Also: Mathod und Holligen gingen andere Wege. Um so schöner war es für Haller, als er gegen Ende seiner Amtszeit als Salzdirektor aus eigenen Ersparnissen eine Herrschaft errang. Sie lag in der Waadt, die damals die Rolle eines Landvorrates spielte wie im 19. Jahrhundert etwa Brasilien. Sein Kauf betraf eine der abgelegensten und kleinsten Gemeinden; aber er durfte sich von 1764 an als Herrn von Goumoens-le-Jux und Eclagnens bezeichnen.

Seine anderen Titel waren in Bern ungültig. Der Kaiser hatte 1749 dem Göttinger Professor ein Adelsdiplom ausgestellt; aber 1731 hatte der Berner Rat beschlossen, daß ausländische Titel in Bern nicht getragen werden durften. «Professor» nannte man ihn ohnehin nicht, und der Titel «Doktor» war gewöhnlich den Stadtärzten vorbehalten. Er blieb «Herr Haller» oder «Herr alt Salzdirektor Haller». Nun durfte er sich «Haller de Goumoens» nennen. Ähnliche Titel wurden in Bern häufig verwendet. Leute gleichen Namens unterschied man damit, so «Sinner de Gessenay», der in Gessenay (Saanen) Landvogt gewesen war, und «Sinner de Ballaigues», der die Herrschaft Ballaigues besaß. Es kam sogar vor, daß der Besitztitel den Namen verdrängte. Eine Familie Wyß, die Mollens besaß, wurde nur noch «de Mollens» genannt.

Die Beiträge Hallers in der Enzyklopädie von Yverdon sind mit HDG (Haller de Goumoens) unterzeichnet und lassen sich dadurch von denen seines ältesten Sohnes Gottlieb Emanuel unterscheiden ${ }^{2}$.

Haller setzte seinen Herrschaftstitel allen anderen voran. In den fünf ersten Bänden der «Elementa physiologiae corporis humani» steht auf dem Titelblatt bei seinem Namen lateinisch: Präsident der Königlichen Gesellschaft der Wissenschaften zu Göttingen, Mitglied der wissenschaftlichen Gesellschaften von Paris, Berlin, Schweden, Bologna, Großbritannien, Upsala und in der Schweiz, Mitglied des bernischen Rates der Zweihundert. In den Bänden 6 bis 8, die 1764-1766 erschienen sind, taucht als erste Zeile unter «auctore Alberto v. Haller» neu auf: «Domino in Goumoens le Jux, et Feudi (in) Eclagnens (vom Herrn in Goumoensle-Jux und des Lehens in Eclagnens; «feudum» heißt Lehen). Die kurzgefaßten «Primae lineae physiologiae» von 1747 erhalten in späteren Auflagen die Beifügung: Domini in Goumoens le Iux et Eclagnens. In den zehn Bänden der Bibliothecae steht «Domino in Goumoens le Jux et (Feudi in) Eclagnens» in größerem Druck als die übrigen Titel. Selbst in den Gedichtausgaben fehlt die Bezeichnung nicht.

Als Haller 1764 zum Mitglied der Holländischen Gesellschaft der Wissenschaf- 
ten in Haarlem ernannt wurde, fragte ihn der Sekretär van der Aa, welche Titel seinem Namen auf dem Diplom beizufügen seien. Haller antwortete am 19. Juli französisch: «Albrecht von Haller, Mitglied des Großen Rates der Republik Bern, alt Vizegouverneur von Aigle und Direktor der Salzwerke der Republik, Seigneur von Goumoens-le-Jux und des Lehens (du fief) von Eclagnens. Präsident der Königlichen Gesellschaft der Wissenschaften zu Göttingen, Mitglied mehrerer Akademien ${ }^{3}{ }^{3}$

Über den Vorgang der Erwerbung erhalten wir Aufschluß aus den Briefen Samuel Engels an Haller. Paul Pulver hat ihren Inhalt in seinem Buch über Samuel Engel zusammengefaßt ${ }^{4}$.

Engel war ein Vetter von Hallers Mutter. Er wirkte vom Oktober 1760 bis Herbst 1765 als Landvogt in Echallens, das deutsch Tscherliz hieß. Savoyen hatte nach den Burgunderkriegen von 1476 die vier Vogteien Murten, Grandson, Orbe und Echallens an Freiburg und Bern abgetreten. Abwechslungsweise wurden sie je fünf Jahre von bernischen und freiburgischen Landvögten verwaltet. Orbe war protestantisch, die anderen drei protestantisch und katholisch gemischt; der Gottesdienst wurde für beide Bekenntnisse in den gleichen Kirchen gehalten. Ein Landvogt von Echallens mußte sowohl die Regierung von Bern wie jene von Freiburg und die Anhänger beider Religionen zufriedenstellen. Das deutsche Bernbiet war reich, das waadtländische arm. Viele Felder waren verwahrlost, versumpft, die Wege schlecht. In den Wäldern wurde fuderweise $\mathrm{Holz}$ gestohlen und nach Lausanne geführt. Alkohol und Müßiggang suchten die Bevölkerung heim. Samuel Engel trachtete die Wohlfahrt zu heben. Er berichtete über die Wälder, leitete Anbauversuche auf einem Musterfeld, kämpfte gegen die Trunksucht und wollte die schlechte Gemeindeverwaltung ordnen. Der Freiburger Ratsherr Nicolas Rämy (1696-1787), der in Freiburg einen Zweig der ökonomischen Gesellschaft gründete, war Engels Gesinnungsfreund.

Als Haller 1763 seine Ersparnisse in einer Besitzung anlegen wollte, suchte er Engels Rat. Dieser hatte sich eben entschlossen, sein eigenes Rebgut in Boudry am Neuenburgersee zu verkaufen, und bot es für 13000 Fr. an. Als größeren käuflichen Besitz bezeichnete er die Herrschaft Goumoens-le-Jux nördlich Echallens für 60000 Fr. zuzüglich die Handänderungsgebühren. Die Besitzerin, Madame de Mollens, sei Witwe geworden und wolle sich auf ihren Familienbesitz zurückziehen.

«De Mollens» nannte sich eine Berner Familie Wyß, die 1790 ihren Namen in «von Weiß» änderte. Der reiche Berner Schultheiß Johannes Steiger (1518-1581), der durch seine Frau, Magdalena Nägeli, ein Schwiegersohn des Eroberers der Waadt war, besaß unter anderen Herrschaften auch jene von Mollens. Sie ge- 


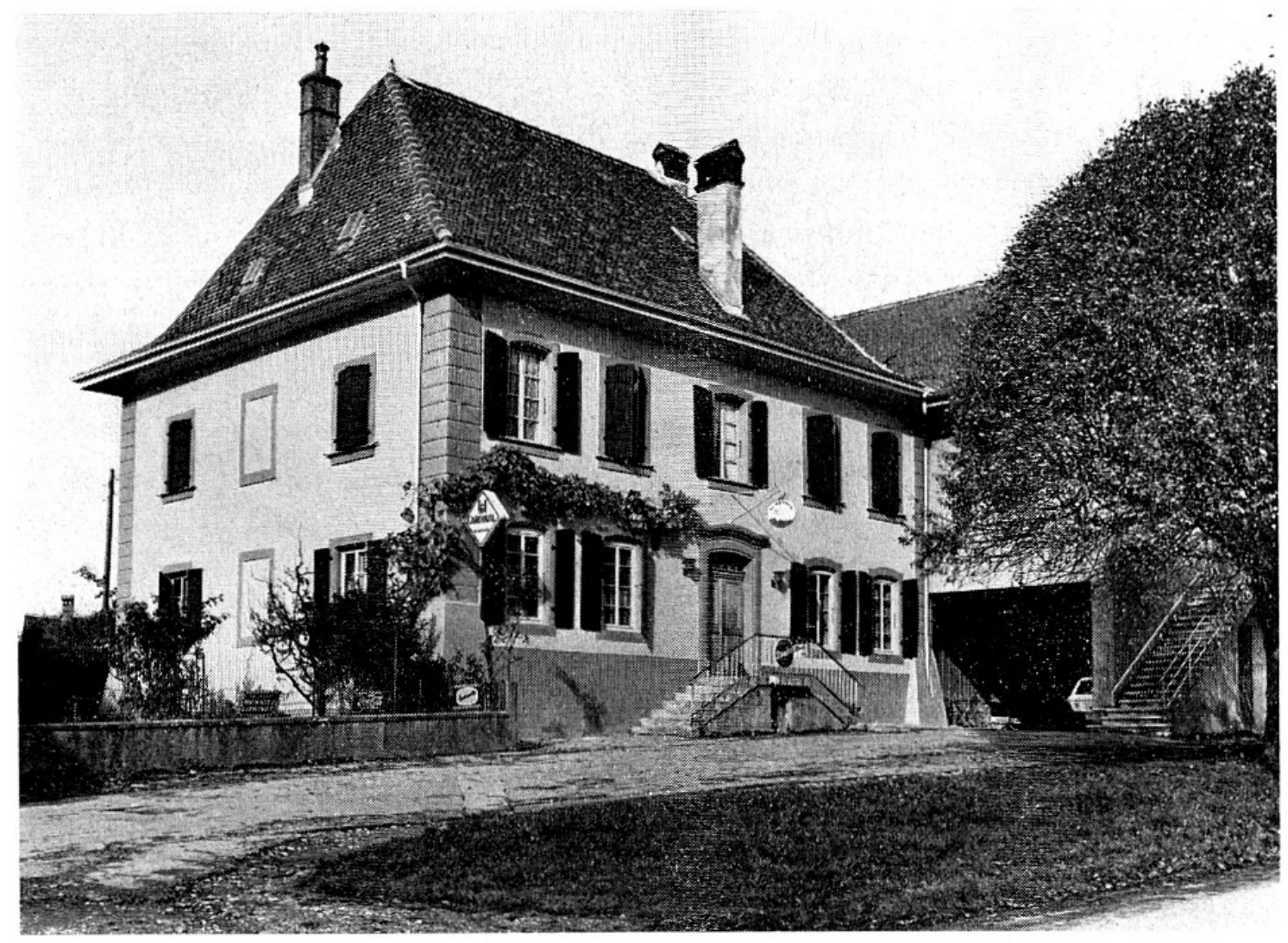

Abb.1. Das Haus des Herrn von Goumoens-le-Jux und Eclagnens. Es steht in Goumoensla-Ville und ist heute Wirtschaft zum Merinoschaf. Aufnahme von Gerhard Howald

langte über seinen gleichnamigen Sohn und Enkel an die Urenkelin Johanna, die 1665 Gabriel Wyß heiratete. Dieser wurde 1660 Ratsherr, 1668 Landvogt von Lausanne, 1678 Salzdirektor und nach dem Tode seines Schwiegervaters 1674 Herr zu Mollens. Als er 1684 starb, fiel Mollens an seinen Sohn Sigmund (1666 bis 1724). Ihm folgte dessen Sohn Bernhard (1690-1755), der sich 1733 mit Salome von Gingins-Chevilly vermählte. Die Tochter Katharina erbte Mollens und heiratete 1760 Alexander von Wattenwyl. Neben Mollens hatte Bernhard Wyß die kleine Herrschaft Goumoens-le-Jux besessen, die nun veräußert werden sollte. Sie bestand aus gutem Land und Wald, einer Mühle und Wirtschaft, der Lehensherrschaft über das Dorf Eclagnens und der Hälfte des Zehntens von Goumoensla-Ville. In dieser Ortschaft stand auch das Herrschaftshaus. Im übrigen gehörte Goumoens-la-Ville der Familie de Goumoëns, die seit 1652 das Berner Burgerrecht besaß. Das kleine Teilgebiet von Goumoens-le-Jux hatte sich einst von der Herrschaft dieses Hauses abgespalten.

Engels Geständnis, daß er das Angebot von Goumoens-le-Jux als einmalige Gelegenheit betrachte und es selber erwerben möchte, wenn er Geld hätte, mußte 
Hallers Kauflust anfachen. Um seine eigene Neigung zu überwinden, ließ Engel sich die eifrige Vermittlung an Haller angelegen sein. Er schrieb ihm vom Sommer 1763 bis Frühling 1764 Brief auf Brief ${ }^{5}$.

Haller konnte sich anfangs nichts vorstellen. An seinen Lausanner Freund, den Arzt Auguste Tissot, richtete er daher am 11. Juli 1763 die Frage: «Man hat mir einen Landbesitz Goumoens-le-Jux vorgeschlagen. Wissen Sie, was das für ein Land ist ? Das Geschäft scheint ziemlich günstig, obschon der Kaufgegenstand ein wenig groß ist. ${ }^{6}$ Tissot war ahnungslos, wollte sich aber erkundigen.

Engel teilte am 28. Juli $1763 \mathrm{mit}$, er sei in Goumoens-la-Ville gewesen und habe ein wenig das fragliche Herrenhaus und seinen Umschwung geprüft. «Das Haus scheint wie neu, da es erst einige Jahre vor dem Tode des Herrn de Mollens erbaut worden ist. Es hat sechs Fenster in der Front und zwei Stockwerke. Zum ersten steigt man über sechs Tritte. Es hat einen schönen Keller und einen Pferdestall, der von der sehr großen Scheune unabhängig ist. Außer dem unbebauten Land ringsum, das für beliebigen Gebrauch freisteht, hat es ein ungefähr drei Posen großes Luzernenfeld, Gärten und Hanf, so daß der dafür festgesetzte Preis von 3750 Franken sehr niedrig ist. ${ }^{7}$

Am 18. August meldete Engel, daß ein Herr de Monnaz mit dem Verkauf von Goumoens beauftragt sei. Die Frage Hallers, ob das Land nicht wild und rauh sei, beantwortete Engel im gleichen Brief mit dem Hinweis: «Wenn Sie alles Land als rauh (sauvage) betrachten, das nicht so mild ist wie das, das Sie gegenwärtig bewohnen, so ist die ganze Schweiz rauh - aber nur vergleichsweise. Goumoens und unser ganzes Land hierherum ist nichts weniger als wild; es ist so fruchtbar, wie man es sich nur wünschen kann, fast wie Rebgärten (aux vignes près). »

Ende August schrieb Haller an Tissot, er müsse nun den Kauf von Goumoens abschließen. Vorher wollte er im September eine Besichtigung vornehmen. Tissot hoffte, Haller auf der Durchreise in Lausanne zu sehen. Er glaubte irrtümlich, es handle sich um Land der Familie de Goumoëns, und er äußerte Bedenken, ob beim Kauf nur eines Teils die Mitherrschaft möglich sein werde. Haller antwortete ihm am 2. September, seine Verkäuferin sei Madame de Mollens. Er habe von ihr Bescheid erhalten. «Wir stimmen nicht ganz überein. Ich werde gleichwohl das Land besichtigen gehen.»Am 10. September gab er noch genauere Auskunft: «Herr de Goumoëns von Goumoens ist Herr von Goumoens-la-Ville. Frau de Mollens besitzt in diesem Goumoens-la-Ville ein Haus mit Gerichtsbarkeit und die Hälfte des Zehntens. Die Lehen von Eclagnens und von Goumoens-le-Jux gehören ihr selber, und es ist Frau de Mollens, von der ich das Land kaufen möchte.» Am 4.Oktober erwähnt Haller in einem Brief an Johannes Geßner in Zürich, daß er inzwischen das Landgut besucht habe ${ }^{8}$. An Tissot berichtet er am 


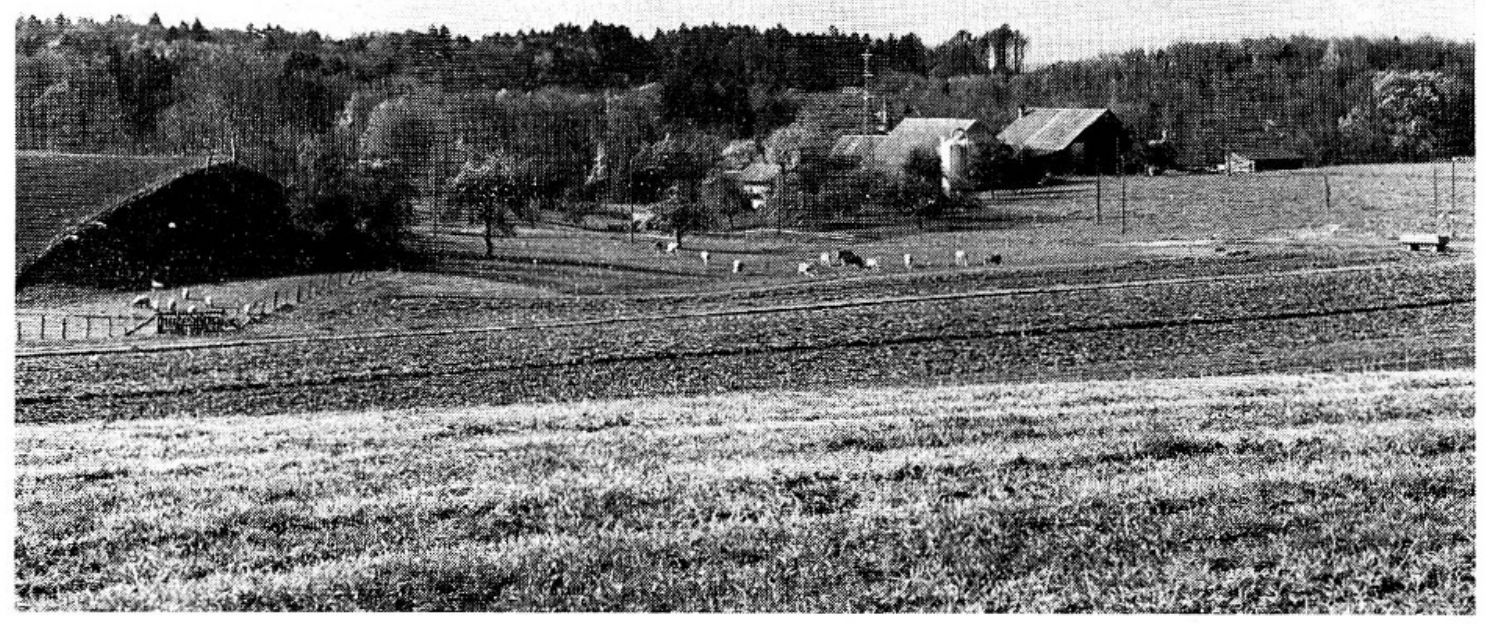

Abb. 2. Blick in die Mulde von Goumoens-le-Jux. In der Tiefe hinter dem Waldkamm fließt der Talent

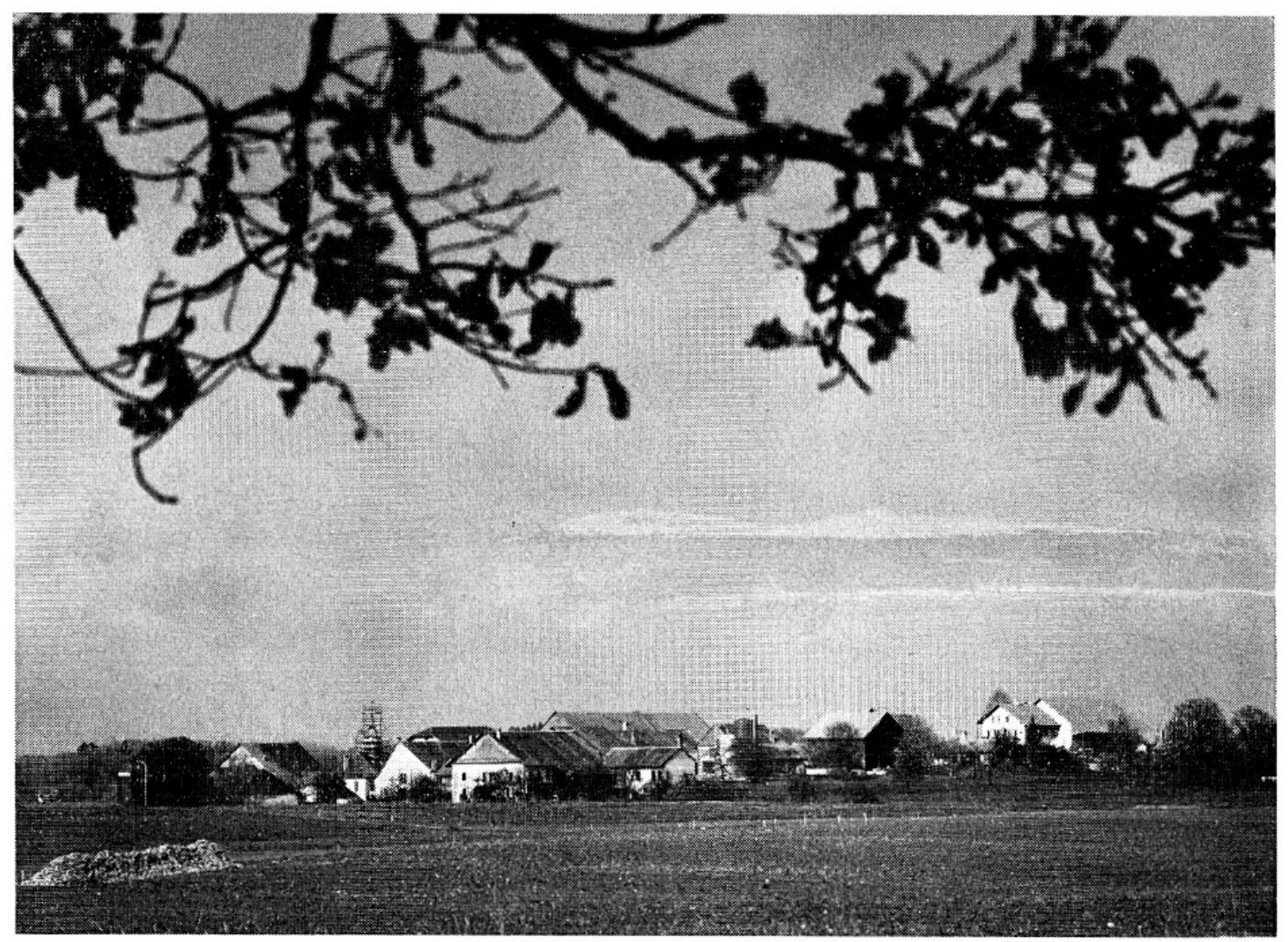

Abb. 3. Die Bodenwelle von Eclagnens. Beide Aufnahmen von Gerhard Howald, Bern 
14. Oktober: «Die Angelegenheit Goumoens stockt, teils wegen Frau de Mollens, die sich nicht entscheidet, teils weil ich noch nicht Maßnahmen für die künftige Verwaltung habe treffen können. Wenn sich diese Dame nur ein wenig der Sache annimmt, so kommt sie zustande. " Am 1. November fragte Tissot, ob Haller den Kauf abgeschlossen habe. Hallers Antwort vom 11. November lautet: «Ich habe Goumoens-le-Jux erworben und soll auf 1.Januar in dessen Besitz gelangen» (J'ai acheté Goumoens le Jux, et dois en etre mis en possession le $1^{\mathrm{r}}$ de Janvier). Tissot beglückwünschte Haller und hoffte, ihn fortan wenigstens einmal im Jahre zu sehen.

Auch an Geßner schrieb Haller am 6.Dezember 1763: «Endlich habe ich Goumoens-le-Jux und Eclagnens um einen recht teuren Preis (satis caro pretio) erworben. Trotzdem sagt man mir, ich hätte mein Geld nicht besser anlegen können. Der Ort ist nicht warm, hat aber für den Getreidebau ausgezeichnete Hügel. Er liegt mitten zwischen Yverdon und Lausanne. Wir sind zusammen just durch jene Gegend gewandert, ohne daß ich damals ahnte, daß sie einmal mir gehören werde (quem non credebam meum fore). » Haller denkt dabei an die gemeinsame Alpenreise von 1728. Etwas später trug er nach: «Meine Dörfchen heißen Goumoens-le-Jux und Eclagnens. In Eclagnens gehören mir nur die Lehen und Zehnten und Bodenzinse.»

Bevor jedoch das Jahr zu Ende ging, spitzte sich der Handel dramatisch zu. Schon lange drohten im Hintergrund die hohen Handänderungsgebühren, und da Haller sich ohnehin überkauft hatte, vergrößerten sie seine Schuldenlast. Die Verhältnisse waren so verwickelt, daß auch Engel sie nicht übersah, wie er am 4. Dezember zugab. Teils waren die Gebühren an Freiburg, teils an Bern und teils an den Herrn von Goumoens-la-Ville zu entrichten. Engel hatte umsonst um Ermäßigung nachgesucht. Auch Frau de Mollens war nicht bereit, den vierten Teil zu übernehmen.

Bei seiner Besichtigung vom September konnte sich Haller nicht recht begeistern. Er hätte gerne auf den Wald und auf Eclagnens verzichtet. Aber als Engel sich anerbot, diese Teile abzukaufen, reute es ihn doch wieder, und er schloß auf das Ganze ab. Den Wald wollte er einer der umliegenden Gemeinden weiterverkaufen. Allmählich wurde die Frage nach den Gebühren brennend. Haller befahl Engel, nichts vorzunehmen, bis alles geregelt sei. In wachsender Erregung fragte er ihn, warum er nichts tue. Engel antwortete mit einem Brief von 16 Seiten. Er habe sich an die Weisung gehalten. Obschon er sich bereits durch Herrn Renier bei den Gemeinden wegen des Waldverkaufs erkundigt habe und in eine Unterhandlung getreten sei, habe er sich zur Nachricht genötigt gesehen, daß alles aufgeschoben sei. Auch teilte er mit, Frau de Mollens habe be- 
reits den Holzschlag von etwa 20 Posen verkauft. Dieses Waldstück sollte vorderhand der Nutzung verschlossen bleiben, bis es sich erholt habe. Dasselbe werde mit den andern Teilen der Fall sein, nach Maßgabe, wie sie ausgebeutet worden sein würden ${ }^{9}$.

Haller verkaufte dann den Wald doch nicht, weil er die Hoffnung faßte, durch Holzhandel allmählich die Geldschulden abtragen zu können. Die hohen Handänderungsgebühren versetzten Haller in Aufruhr. Durch ein Mißverständnis glaubte er, Engel sei daran schuld. Dieser wehrte sich: «Es ist verdrießlich, daß ich mich nicht verständlich machen kann. Wann habe ich denn gesagt, daß ich soviel und nicht weniger verlange? Habe ich nicht vielmehr klar dargelegt, daß es nicht an mir liegt, die Last zu bestimmen, sondern an den Generalkommissären? ${ }^{10}$ Er habe eine Berechnung anzustellen versucht, die Haller gerecht fände, wenn er nicht voreingenommen wäre. Den Wald könne man nicht unter 10000 Franken veranschlagen. Er biete sie ihm auch jetzt noch dafür an. Am Schluß versichert Engel, er habe ihn so beraten, wie wenn er es für sich selbst getan hätte (que je vous ay conseillé comme pour moy même).

Auch am 10. Januar 1764 klagt Engel: «Es ist sehr betrüblich für mich, daß Sie trotz dem ganzen Eifer, mit dem ich mich für Sie verwendet habe und wovon jedermann hier und in Freiburg mir Zeugnis geben wird, alles im gegenteiligen Sinne aufgefaßt haben, nicht nur in Ihren Briefen an mich, sondern indem Sie Ihre Klagen sogar nach Bern ausbreiten.» ${ }^{11}$ Er gestehe ihm, daß er über die Art verlegen sei, wie er sich in Zukunft benehmen solle. Seine Freundschaft habe ihn Pläne zu Hallers Gunsten machen lassen; aber es sei unangenehm, daß das Mißtrauen es nicht merke und das Gegenteil annehme. Engel wirft Haller vor, daß er weder die Gebräuche dieser Gegend noch die Art der Leute kenne. Nun wolle er ihm Einfälle, nicht Ratschläge unterbreiten. Wenn sie ihm nicht gefielen, solle er sie streichen und vergessen, ihm aber nicht schlechte Absichten zuschreiben. Haller sei erst dann Besitzer, wenn die Urkunde beglaubigt sei, und bis dahin habe er kein Recht, in irgendeiner Weise zu handeln. Die höflichen Erklärungen des Herrn de Monnaz, wie er den Auftrag zu schätzen wisse, seien von der gleichen Art wie die der Päpste, wenn sie Königreiche vergaben, die ihnen nicht gehörten. In einem Nachsatz bemerkt er: «Ich habe mich soeben mit jemandem aus Goumoens unterhalten, der den Wald für ein Kleinod hält, ganz wie ich selber, und er ist überrascht, daß Sie nicht wenigstens 6 bis 8 Posen für den Notfall behalten wollen. ${ }^{12}$

Haller hatte den Vertrag noch nicht regeln lassen, weil er auf eine Antwort von Herrn de Goumoëns wartete. Bald bereute Haller seine Vorwürfe. Er bat Engel, nicht weiter von der Handänderungsgebühr zu sprechen, und dieser ant- 
wortete am 16. Januar: "Sie haben recht, und ich tue mein möglichstes, um alles zu vergessen, was in dieser Beziehung vorgefallen ist. ${ }^{13}$ Engel schützte Haller vor Fehltritten und löste das Gewirr der Rechtsverhältnisse, so daß der Kauf zu gutem Ende kam. Am 14. Februar 1764, schickte Steck die beglaubigte Kaufsurkunde aus Bern an Haller nach Roche: «Cy joint l'acte d'aquis... » Schon am 17. Februar beglückwünschte ihn Nicolas Rämy aus Freiburg, und tags darauf schrieb der Baron Dalt de Tieffenthal in Freiburg einen Glückwunschbrief.

Die Freude überwog nun; aber Haller mußte sich auf große Abzahlungen rüsten. Er hatte nicht gerne Schulden und erwog hin und her, wie er zu Geld kommen könnte. Aus der Herrschaft flossen ihm jährliche Abgaben in Getreide, Wachs und Geld zu; das Getreide konnte er verkaufen. Vor allem aber wollte er nun den Wald ausbeuten und eine Holzhandlung in Lausanne einrichten. Am 24. Februar 1764 schrieb er an Tissot, heute beendige er die Niederschrift seiner achtbändigen Physiologie, die er 1751 begonnen habe. Nur noch die Vorworte und Schlußergänzungen fehlten. «Es ist lächerlich, mit Ihnen über Holz und einen Holzplatz zu sprechen.» Er habe berechnet, jährlich 100 oder $200 \mathrm{Ma}$ (moules) Holz in Goumoens schlagen zu lassen. Um nicht zu einem Verkaufspreis genötigt zu werden, habe er im Sinn, selber einen fixen Preis zu bestimmen. «Ich möchte (in Lausanne) einen Platz mieten, um es zu lagern, und jemanden haben, der es verkauft.» Für seine Mühe solle der Händler für jedes Maß eine feste Entschädigung erhalten. Tissot berichtete am 4. März über seine Bemühungen, einen geeigneten Mann und einen Platz für Hallers Holzhandlung zu finden. Haller antwortete am 9. März mit Ausführungen über Holzmaße. Er vergleicht das Lausanner Maß mit dem von Goumoens ${ }^{14}$. Haller glaubt, daß nur ein Engroshandel sich lohne. Man werde das vorsichtig einfädeln müssen, sobald sein Förster nächste Woche in Goumoens eingetroffen sein werde. Nach und nach richte sich Goumoens ein. Wenn der Holzhandel gelinge, werde er einen guten Kauf gemacht haben. Er habe einen sehr guten Förster. Die Pinte und die Mühle habe er wieder verpachtet. Ein Einnehmer werde ihm die Zinsen einziehen.

Dem von Roche nach Goumoens aufbrechenden Förster gab Haller am 12. März ein Bücherpaket mit. Er sollte es bei Wilhelm Otto Struve in Ouchy abgeben, weil einige der Bücher diesem gehörten. Die für Tissot bestimmten Bücher seien ebenfalls in dem Paket enthalten.

Haller plante für Mitte April einen eigenen Aufenthalt von drei oder vier Tagen in Goumoens, um das Nötigste anzuordnen. Den Holzverkauf in Lausanne übernahm ein Herr Trachsel. Haller bat Tissot am 25. Juni, mit Trachsel zu sprechen, der pro Maß nur 19 statt 20 Franken für das nach St-François gelieferte Holz zahlen wolle. Tissot mußte melden, Trachsel gebe nicht nach und verlange die 
Zusicherung, daß Hallers Holz einzig an ihn gehen werde. Er wollte es jeweilen bei der Ablieferung ausmessen. Haller antwortete am 29. Juni, dies sei wegen des Zeitaufwandes fast undurchführbar, da seine Leute von Goumoens bis Lausanne eine starke Tagereise hätten. Daher denke er an einen Ausweg. Trachsel könnte zweimal jährlich das Holz in Goumoens messen. Oder man könnte den drei Transportwagen einen bestimmten Rauminhalt geben. Zur eigenen Sicherheit sollte Herr Trachsel nach freier Wahl ein- oder zweimal im Monat die Wagen in Gegenwart der Leute von Goumoens in Lausanne nachmessen.

Am 1. Juli entrichtete Haller eine Abzahlung von 12000 Franken an Madame de Mollens. Sein Amt als Salzdirektor in Roche dauerte noch bis Ende September. Am 14. September 1764 schrieb er aufgeregt an Tissot, in der Amtskasse fehlten 2500 Franken. Er solle ihm in Lausanne einen Gläubiger suchen, der den Betrag vorschieße. «Dieses Abenteuer greift mich an, erhitzt mich und raubt mir den Schlaf, weil mir nur wenig Zeit übrigbleibt, um die Ursache dieses Fehlbetrags abzuklären. Wenn ich dieses Unglück geahnt hätte, so hätte ich am 1.Juli an Frau de Mollens nur 10000 statt 12000 Franken bezahlt. » Drei Tage später konnte er Tissot beruhigt mitteilen, es handle sich um einen Rechnungsfehler. Statt 2500 sollte es 250 heißen. Er könne diesen Betrag decken; Tissot möge sich nicht weiter bemühen.

Am 26. September erfolgte die Abreise. Haller hatte zuerst einen Besuch bei Tissot vorgesehen, schrieb aber am Tag vor dem Aufbruch: «Wir sind zuviel Leute und Angestellte, um Sie diesmal zu belästigen. Ich verspare dieses Vergnügen für nächsten Frühling in der Hoffnung, dann von Goumoens nach Lausanne zu gehen. «Wir sind in so großem Durcheinander, daß ich nicht mehr weiß, was ich tue ... Alles ist auf einmal gekommen. » Unterwegs weilte er kurz in Goumoens.

Im Mai 1765 beklagte er sich, daß Herr Trachsel ihm nicht antworte, und im Juni schrieb er aus Bern an Tissot: «Eine meiner Sorgen ist dieses Goumoens. Ich suche einen Pächter, dem ich es anvertrauen kann und mit dem ich den Ertrag zu teilen bereit bin.» Er bittet um einen Vorschlag. Das Holz werde er fortan wagenweise verkaufen.

Anfang Juli 1765 fragte Tissot, wann Haller nach Goumoens reisen werde. Haller antwortete, er werde es anzeigen. Aber im August meldete er, er behandle den kranken Schultheißen Johann Anton Tillier und könne erst kommen, wenn dessen Gesundheit es erlaube.

Darüber wurde es 1766. Am 25. April teilte Haller mit, er gehe im Mai nach Goumoens und würde sich freuen, Tissot dort zu sehen. Im Mai schrieb Haller, eine Unpäßlichkeit habe die Reise verschoben, aber nicht aufgehoben. Er arbeite am Pflanzenbuch. Nun werde er Ende Juni kommen. Er könne zusammen mit 
Emanuel Hartmann reisen, der als neuer Landvogt in Nyon antrete. Jedoch am 20. Juni gestand Haller, er habe keine freie Zeit. Man sei jetzt in Bern mit den Genfer Angelegenheiten beschäftigt. Er komme bis in einem Monat. Tissot wollte ihn in Goumoens besuchen. Endlich hoffte Haller am 3. August, am Dienstag oder Mittwoch über Payerne nach Goumoens reisen zu können. «Ich wage fast nicht mehr davon zu sprechen, da ich schon so oft genötigt war, anderen Sinnes zu werden. »

Aus dem Jahre 1767 ist in den Briefen an Tissot nur eine Erwähnung vom 25. Juni enthalten. Die Genfer Vorfälle beanspruchten ihn täglich mehr, und seine Studien litten darunter. «Kennen Sie einen Wirt Vernand von Lausanne, der sich um die Schenke von Goumoens bewirbt? Kann man ihm diese Pacht anvertrauen?» Die Auskunft über das Ehepaar Vernand lautete günstig.

Tissot fragte immer etwa wieder, ob Haller nicht nach Goumoens kommen werde. Endlich, 1768, war es so weit. Ende April versprach Haller, er werde bald kommen und ihn auf der Durchreise benachrichtigen. Tissot freute sich. Am 12. Juni versicherte Haller, er werde kommen, müsse aber zuerst einen Schnupfen ausheilen lassen, der seine Brust beenge. Tissot bat, ihm einige Bücher nach Goumoens mitzubringen, vor allem Anton de Haens elften Band der «Ratio medendi». Haller kündigte seine Ankunft für Sonntag, den 14.August, an. Auch an Johannes Geßner berichtete er, nächste Woche werde er das Landgut in Goumoens aufsuchen, denn diese Reise könne nicht länger aufgeschoben werden; aber sie werde nicht lange dauern. Ein weiterer Brief gab Tissot die Bestätigung, er breche nun wirklich am 13. auf und werde bis am 18. oder 19. in Goumoens wohnen. «Diese kleine Reise verwirrt mich sehr. Ich tauge nur noch für das Studierzimmer und die Lektüre. Es wird wohl die letzte Reise sein, die ich nach Goumoens unternehme. Die Sorge dafür will ich meinem ältesten Sohn übergeben, der Goumoens auch nach meinem Tode besitzen soll. »15

Leider konnte Tissot Haller nicht treffen. Als er am Montag eben das Pferd bestiegen hatte, um nach Goumoens zu reiten, brachte ein Bote die Nachricht, sein Vater sei am Abend zuvor an Lähmungserscheinungen erkrankt. Als Arzt stand er an diesem und an den folgenden Tagen seinem Vater bei, der am 4. September starb.

Haller kehrte nie mehr in sein Besitztum zurück. Nach der ersten Besichtigung vom September 1763 hat er es wohl nur im April und September 1764 und im August 1768 kurz gesehen, insgesamt etwa zwei Wochen. Die Zeit reichte nicht aus. Es hat etwas Rührendes, daß Haller ein so schönes Ferienhaus besaß und eigentlich nie dort war. Nur unter solchen Opfern konnte das überragende Lebenswerk entstehen. 
Die späteren Nachrichten sind spärlich. An Geßner schreibt Haller Ende 1770, er habe sein Getreide, die Abgabe von Goumoens, günstig verkauft, und ebenso habe das Zehntengeld die Schätzung fast um das Doppelte übertroffen. Auch im Herbst 1771 erwähnt er gegenüber Geßner das Getreide, das aus dem Landgut herbeigeholt werden müsse.

Am 1.Juni 1771 schickte Tissot Bücher an Haller. Der Überbringer, Herr Dufour aus Goumoens, wollte Hallers Wohnung dort mieten. Tissot empfahl ihn.

Ein letztes Mal erwachte in Haller der Wunsch, Goumoens wiederzusehen, als er sich gegen Ende August 1775 von einer Erkrankung erholt hatte. Wieder hoffte Tissot, ihn besuchen zu können. Aber die Reise kam nicht zustande. Am 6.Oktober konnte Haller nur melden, er habe Herrn Penier in Goumoens geschrieben, er solle Tissot jährlich weiterhin 3 Louisdor bezahlen. Penier war Hallers Einnehmer. Er stammte aus Echallens und hatte 1769, eine Schwindsucht befürchtend, auf Hallers Wunsch Tissot um Rat gefragt ${ }^{16}$.

Haller konnte die Herrschaft nie ganz abzahlen. In Bern war seine Besoldung allzu gering; der Holzhandel hatte wenig eingetragen, und weitere Geschäfte unternahm Haller nicht. Einer seiner Söhne, Rudolf Emanuel, ergriff die kaufmännische Laufbahn. Als Angestellter des Hauses Hornecca in Amsterdam begann er zu spekulieren. Der Vater mahnte ab und schrieb ihm am 29. Juli 1769, Börsengeschäfte seien gefährlich wie ein stürmisches Meer. Rudolf habe ja keine eigenen Mittel. «Selber habe ich Goumoens, eine Liegenschaft im Wert von 100000 Franken. Ich schulde daran 21000 Franken. Meine ganze Spekulation beschränkt sich darauf, diese Schuld zu vermindern» (Pour moi, j’ai Goumoens, effet de 100000 livres. Je dois là-dessus 21000 francs. Toute ma spéculation se réduit à diminuer cette dette $)^{17}$.

Haller war 14 Jahre «Herr von Goumoens-le-Jux und Eclagnens». Daß er sich darüber freute, gab der Erwerbung ihren Sinn. Keiner der Söhne und Schwiegersöhne wollte sie nach Hallers Tode übernehmen. Gottlieb Emanuel machte zwar ein Angebot. Als die anderen es ablehnten, zog er sich beleidigt zurück. Goumoens wurde zum Verkauf angeboten, fand aber keinen Abnehmer. Gottlieb Emanuel führte für die Witwe Hallers die Verwaltung weiter. Er starb zu Ostern 1786 ${ }^{18}$. Reich geworden, übernahm der Bruder Rudolf Emanuel Goumoens-le-Jux, kümmerte sich aber, als Bankier in Paris lebend, nicht darum. Er glaubte an die Zukunft Frankreichs, wollte dort bleiben und nicht in die Schweiz zurückkehren. 1791 bat er seinen Schwager, den Berner Bankier Ludwig Zeerleder (1727-1792), die Veräußerung von Goumoens-le-Jux zu beschleunigen. Im Januar 1792 hatte Zeerleder einen Käufer gefunden. Leicht wurde dahingegeben, was der Vater schwer errungen hatte. 


\section{Anmerkungen und Quellenhinweise}

${ }^{1}$ Holligen blieb in der Familie. Gabriel Herborts einzige Tochter Anna Margaretha heiratete 1759 Johann Rudolf Mutach (1729-1793). Er wurde 1783 Herr des Schlosses. Nach seinem Sohne Karl Ludwig übernahm es der Sohn von dessen Vetter, Arnold Ludwig Mutach (1801-1852), dann dessen Sohn Vincent Arnold (1829-1911, Eisenbahningenieur). Dieser hatte drei Söhne und eine Tochter Hilda. Zwei der Söhne, Ludwig Alfred und Daniel, verkauften ihre Anteile dem Bruder Alois (1875-1946, Arzt, ledig). Ihn überlebte in Holligen die gleichfalls ledige Schwester Hilda (1870-1955). Der Bruder Daniel (Architekt) starb 1948 kinderlos; Ludwig Alfred hinterließ eine einzige Tochter, Marie Katharine (geboren 1904), die 1925 den Fürsprecher Walter von Werdt heiratete. Diesem Ehepaar von Werdt-von Mutach vermachte Hilda Holligen.

Für alle Familienangaben siehe: Bernhard von Rodt (1892-1970), Genealogien burgerlicher Geschlechter der Stadt Bern, 6 Bände, 1950 (handschriftlich in der Berner Burgerbibliothek).

2 Erich Hintzsche, Albrecht von Hallers Tätigkeit als Enzyklopädist. In: Clio Medica 1 (1966), darin S. 251.

${ }^{3}$ Siehe: Gerrit A.Lindeboom, Sechs Briefe Albrecht von Hallers. In: Gesnerus 33 (1946), Heft 3/4, darin S. 212.

4 Paul Pulver, Samuel Engel. Ein Berner Patrizier aus dem Zeitalter der Aufklärung, 1702-1784. Bern 1937 (Kapitel: Als Landvogt in Echallens, 1760-1765, S.159-183, besonders S.181-183 und Anmerkungen S. 356).

5 Handschriftliche Briefe Engels an Haller in der Burgerbibliothek Bern, Mss. Hist. Helv. XVIII, Bd. 22, 24 und weit mehr in Bd. 52, 53.

${ }^{6}$ Die im Original französischen Briefe Hallers an Tissot können nachgelesen werden in: Erich Hintzsche (Hrsg.), Albrecht von Hallers Briefe an Auguste Tissot, 1754-1777, Bern/Stuttgart/Wien 1977 (mit Register von Urs Boschung). Benützt wurden hier S. 165 f., 171-173, 175 f., 179-182, 184f., 191 f., 194, 206 f., 209 f., 234-236, 238-240, 263 f., 270, 285, 287-293, 334, 428f., 499.

7 «J'ay été à Goumoens, mon cher Cousin, et ay un peu examiné la Maison Seigneuriale en question et ses environs. La Maison paroit comme neuve, ayant été batie Seulement quelques années avant la mort de Mr. De Mollens; elle a 6. Fenetres de front, et 2. Etages; au premier on monte par 6 marches; Il y a une belle Cave, et une Ecurie independante de la Grange, qui est tres grande; outre la Terre vague aux environs, à tout usage, il y a environ 3. poses en Luzerniere, Jardins, Chenevis et de Sorte que le prix de $£ 3750$. qu'on en fixe, est tres bas. » (Nach dem Original in der Burgerbibliothek.) - Das Haus wird auch erwähnt in: Eugène Mottaz, Dictionnaire historique, géographique et statistique du Canton de Vaud, Lausanne 1911. Es entspricht dem heutigen Café du Mérinos. In der Front zählt man nicht sechs, sondern fünf Fenster; aber daß sechs Stufen zum Eingang führen, stimmt immer noch.

${ }^{8}$ Henry E.Sigerist (Hrsg.), Albrecht von Hallers Briefe an Johannes Gesner, Berlin 1923. Darin S. 340, 342f., 416, 445, 458.

9 «Mad. de Mollens a deja vendu la coupe d'environ 20 poses, laquelle piece devroit etre refermée pour se repeupler; et les autres, à mesure qu'elles seroient exploitées.» (Mss. Hist. Helv. XVIII., Bd. 52, Brief 181, letzte Seite.) 
10 "Il est facheux, Monsieur et tres cher Cousin, que je ne puiße me faire comprendre; quand est ce que j'ay dit, que je demandois autant, et pas moins? N'ay je pas dit plutôt clairement, que ce n'est pas à moy à determiner le Laod, mais a Mrs. les Commissaires generaux? » (Brief 183, Beginn.)

11 «Il est tres affligeant pour moy, qu'avec tout le zèle avec lequel je me suis employé pour vous, et dont tout le monde ici et à Fribourg me rendra temoignage, vous avez pris tout en contre Sens, non seulement dans vos lettres à moy, mais en repandant vos plaintes à Berne même.»

12 «Je viens d'avoir un entretien avec quelqu'un de Goumoens, qui regarde le bois comme un bijou, tout comme moy, et il est surpris, que du moins vous n'en vouliez pas conserver 6 à 8 poses pour les cas de besoin.»

13 «Vous ne voulez pas que je parle davantage du Laod; vous avez raison, je fais mon possible pour oublier tout ce qui s'est passé à ce sujet.»

14 Siehe Hintzsche, Hallers Briefe an Tissot, S.180. So wie Alfred G. Roth (Alpfahrt in der Schweiz, Burgdorf 1976; darin: Eine Maß Milch, S. 20-26) das Milchmaß bei Haller untersucht hat, könnte ein Fachmann hier über das Holzmaß bei Haller berichten.

15 «Ce petit voyage me derange beaucoup. Je ne vaux plus rien que pour le Cabinet et la lecture. Ce sera le dernier que je ferai peutetre a Goumoens, dont je donnerai le soin a mon fils ainé, qui egalement en sera le possesseur aprez ma mort.» (Hintzsche, Hallers Briefe an Tissot, S. 292; Brief vom 11. August 1768.)

16 Hintzsche, Hallers Briefe an Tissot, S. 312.

17 Albert de Haller, Rodolphe-Emmanuel de Haller, 1747-1833, d'après sa correspondance, Lausanne 1909 (54 S., mit Porträt). Zu Goumoens: S. 6, 12, 15 (Verkauf).

18 Hans Haeberli, Gottlieb Emanuel von Haller. Ein Berner Historiker und Staatsmann im Zeitalter der Aufklärung, 1735-1786. In: Archiv des Historischen Vereins des Kantons Bern 41 (1952); darin S. 277, 287.

\section{Summary}

During his activity as a director of the salt-works at Roche, Haller bought in 1764 the dominion of Goumoens-le-Jux and Eclagnens, two small villages in Vaud (Switzerland). His cousin, Samuel Engel, was the mediator of this purchase. A beautiful house in Goumoens-la-Ville was destined for the master. But Haller had no time to stay there. Because he could not pay the whole sum, he tried to entertain, with the help of his friend Auguste Tissot, a wood-trade in Lausanne. In Haller's letters to Tissot and to Johannes Gessner of Zurich, we find some references to Goumoens. On the title pages of the books which Haller published between 1764 and 1777 , we often read under his name: «Dominus in Goumoens le Jux et Eclagnens ».

Dr.phil. Heinz Balmer

Medizinhistorisches Institut

Rämistraße 71

8006 Zürich 